\title{
CONVEXITY OR CONCAVITY INEQUALITIES FOR HERMITIAN OPERATORS
}

\author{
JEAN-CHRISTOPHE BOURIN
}

\begin{abstract}
Given a Hermitian operator, a monotone convex function $f$ and a subspace $\mathscr{E}$, $\operatorname{dim} \mathscr{E}<\infty$, there exists a unitary operator $U$ on $\mathscr{E}$ such that $f\left(A_{\mathscr{E}}\right) \leqslant U f(A)_{\mathscr{E}} U^{*}$. (Here $X_{\mathscr{E}}$ denotes the compression of $X$ onto $\left.\mathscr{E}\right)$. A related result is: For a monotone convex function $f, 0<\alpha, \beta<1, \alpha+\beta=1$, and Hermitian operators $A, B$ on a finite dimensional space, there exists a unitary $U$ such that $f(\alpha A+\beta B) \leqslant U\{\alpha f(A)+\beta f(B)\} U^{*}$. More general convexity results are established. Also, several old and new trace inequalities of Brown-Kosaki and Hansen-Pedersen type are derived. We study the behaviour of the map $p \longrightarrow\left\{\left(A^{p}\right)_{\mathscr{E}}\right\}^{1 / p}$, $A \geqslant 0,0<p<\infty$.
\end{abstract}

Mathematics subject classification (2000): 47A20, 47A30, 47A63.

Key words and phrases: compressions, convex functions, eigenvalues, invariant norms.

\section{REFERENCES}

[1] R. Bhatia, Matrix Analysis, Springer, Germany, 1996.

[2] J.-C. BouRIN, Total dilation II, Linear Algebra Appl. 374/C (2003) 19-29.

[3] L. G. BROWN AND H. KosAKI, Jensen's inequality is semi-finite von Neumann algebras, J. Operator Theory 23 (1990) 3-19.

[4] C. DavIS, A Schwarz inequality for convex operator functions, Proc. Amer. Math. Soc. 8 (1957) $42-44$.

[5] F. HANSEN, An operator inequality, Math. Ann. 258 (1980) 249-250.

[6] F. HANSEN AND G. K. PEDERSEN, Jensen's inequality for operator sand Lowner's Theorem, Math. Ann. 258 (1982) 229-241.

[7] F. HAnsen And G. K. Pedersen, Jensen's operator inequality, Bull. London Math. Soc. 35 (2003) 553-564.

[8] B. SIMON, Trace Ideals and Their Applications LMS lecture note, 35 Cambridge Univ. Press, Cambridge, 1979.

[9] X. ZHAN, Matrix Inequalities, LNM 1790, Springer, Berlin, 2002. 